\section{From the Centers for Disease Control}

\section{BACTERIAL CONTAMINATION OF PLATELET POOLS, OHIO 1991}

From June 27 through July 30, 1991, four episodes of bacterial contamination of platelet pools occurred in an Ohio hospital and were reported by the hospital through the Food and Drug Administration (FDA) to the Centers for Disease Control (CDC). This report summarizes the results of the epidemiologic investigation of these episodes.

Three of the four patients who received the platelet pools were hospitalized for malignancies and developed chills and rigors within one to 40 minutes after initiation of the transfusion; one of the three developed hypotension. After two of these episodes, the hospital began pretransfusion gram staining and culturing of all platelets but did not delay platelet transfusion awaiting gram stain or culture results. This increased surveillance detected a fourth contaminated platelet pool, but it had already been transfused to a hemodialysis patient with idiopathic thrombocytopenia associated with myelodysplasia; this patient developed asymptomatic bacteremia.

The FDA and CDC initiated an investigation to attempt to detect the source of and risk factors for contamination of these platelet pools. A contaminated platelet pool was defined as a platelet pool with a positive bacterial culture confirmed by either a positive gram stain of the pool, a positive culture in an individual unit that was part of the platelet pool, or a positive culture from blood obtained from the transfusion recipient from June 27 through July 31 . The four contaminated platelet pools, each derived from five individual platelet units, were positive by gram stain and culture for Bacillus cereus (two platelet pools) and Staphylococcus epidermidis and Pseudomonas aeruginosa (one platelet pool each); colony counts ranged from $10^{6}$ to $10^{8}$ colony-forming units (CFU) per $\mathrm{ml}$ in the platelet pools, and from 10 to $10^{10} \mathrm{CFU} / \mathrm{ml}$ among 11 of the 20 individual platelet units constituting the pools.

On average, the individual platelet units constituting the contaminated platelet pools were pooled 2.5 hours (range $=15$ minutes-3.2 hours) before transfusion. Investigators examined data on the four contaminated platelet pools for associations with the phlebotomists, bloodmobile sites or dates, component separation technicians, pooling technician, pooling date and time, and platelet age. However, no units from any two pools shared any of these factors.

From June 27 through July 30, the rate of bacterial contamination of platelet pools at the hospital
(4 [0.4\%] of 1,063 platelet pools transfused) was significantly greater than for the previous 21 months (1 [0.01\%] of 7,350 platelet pools transfused, $p=.001$ ). During the 21 months before June 27, 1991 (when the hospital instituted more stringent guidelines for detection and reporting of platelet-transfusion reactions), the nursing staff at the hospital increasingly reported platelet-transfusion reactions to the hospital blood bank (99\% of which resulted in culturing of the involved platelet pool). The rate of reported reactions, by month, increased from 2.3 per 1,000 platelet pools transfused during September 1989 to 72.4 reported reactions per 1,000 platelet pools transfused during July 1991 ( $\phi<.001$, chi square for linear trend).

The hospital continues pretransfusion gram staining and culturing of all platelets and now requires a negative gram stain before releasing the platelets for transfusion.

\section{MMWR. 1992;41:36-37.}

\section{THE SECOND 100,000 CASES OF ACQUIRED IMMUNODEFICIENCY SYNDROME, UNITED STATES, JUNE 1981- DECEMBER 1991}

The first cases of acquired immunodeficiency syndrome (AIDS) were reported in June 1981. ${ }^{1}$ From 1981 through December 1987, 50,000 AIDS cases had been reported to the Centers for Disease Control, and by August 1989, 100,000 cases had been reported. ${ }^{2}$ From September 1989 through November 1991, state and territorial health departments reported 100,000 additional cases. By December 31, 1991, a cumulative total of 206,392 cases had been reported, and the cumulative number of reported deaths associated with AIDS was 133,232 . This report presents characteristics of the first and second 100,000 persons with AIDS.

Overall, most reported AIDS cases occurred among homosexual/bisexual men (59\%) and injectingdrug users (IDUs) (22\%). Of the first 100,000 reported AIDS cases, 61\% occurred among homosexual/ bisexual men with no history of IDU, and $20 \%$ among female or heterosexual male IDUs. In comparison, of the second 100,000 reported cases, 55\% occurred among homosexual/bisexual men with no history of IDU, and $24 \%$ occurred among female or heterosexual male IDUs.

The second 100,000 cases reflect an increasing proportion of persons with AIDS who have been reported to have had heterosexual exposure to persons at risk for human immunodeficiency virus (HIV) infection. Of the first 100,000 persons with AIDS, 5\% were attributed to heterosexual transmission, compared with 7\% among the second 100,000-a 44\% increase. Of all AIDS cases among women, $34 \%$ were 
attributed to heterosexual transmission, and women accounted for $61 \%$ of all cases attributed to heterosexual transmission. Of the first 100,000 persons with AIDS, 9\% were women, compared with $12 \%$ of the second 100,000 persons. The first 100,000 persons with AIDS included 1,683 children, of whom $81 \%$ were born to mothers with or at risk for HIV infection; the second 100,000 persons with AIDS included 1,702 children, of whom $87 \%$ were born to mothers with or at risk for HIV infection.

A disproportionate number of AIDS cases continues to be reported among blacks and Hispanics. Of the first 100,000 reported cases, $27 \%$ occurred among blacks and $15 \%$ among Hispanics; of the second 100,000 reported cases, these proportions increased to $31 \%$ and $17 \%$ for blacks and Hispanics, respectively.

The proportion of AIDS cases related to transfusion as a mode of exposure declined in both adults (2.5\% to $1.9 \%$ ) and children (11\% to $5.6 \%$ ) from the first to the second 100,000 cases.

\section{REFERENCES}

1. Centers for Disease Control. Pneumocystis pneumonia-Los Angeles. MMWR. 1981;30:250-252.

2. Centers for Disease Control. First 100,000 cases of acquired immunodeficiency syndrome-United States. $M M W R$. 1989;38:561-563.

From the M M WR. 1992:41:28-29. 